\title{
El XIII Plan Quinquenal: antecedentes, contexto, contenidos y expectativas
}

\section{The 13th Five-Year Plan: Precedents, Context, Contents and Expectations}

\author{
Xulio Ríos ${ }^{1}$ \\ Director del Observatorio de la Política China
}

Recibido: 24-12-15

Aprobado: 07-02-16

\section{Resumen}

Este ensayo presenta una contextualización general del momento actual de la economía china, de la importancia histórica de la planificación y su evolución, y de las ambiciones y expectativas que pudiera reflejar el XIII Plan Quinquenal. Su asociación con la revitalización del país y el "sueño chino" y sus implicaciones internacionales confieren a esta estrategia una significación ciertamente crucial para culminar con éxito el largo proceso de modernización de China.

Palabras-clave: Economía china, XIII plan quinquenal, sueño chino, modernización.

\begin{abstract}
This essay aims at presenting a general contextualization of the current economic situation in China, the historical importance of planning and its development, and the ambitions and expectations that the 13th Five-Year plan could reflect. Its association with the revitalization of the country and the "Chinese Dream", and its international implications give a crucial importance to this strategy in order to successfully complete the long process of modernization of China.
\end{abstract}

\footnotetext{
${ }^{1}$ (director@politica-china.org). Xulio Ríos (España, 1958) es licenciado en Derecho y director del IGADI, Instituto Gallego de Análisis y Documentación Internacional. (www.igadi.org). Estudioso de los temas chinos desde hace varios años, es miembro del Consejo Asesor de Casa Asia (España), Director del Observatorio de la Política China (www.politica-china.org), Promotor y Coordinador de la Red Iberoamericana de Sinología. Ha residido de forma permanente en China (2006-2010 y 201314) y fue Visiting Scholar en la Academia Sínica, Taipei, enero a abril 2012.
} 
Key-words: Chinese Economy, 13th Five-Year Plan, Chinese dream, Modernization.

El XIII Plan Quinquenal (2016-2020), aprobado en la quinta sesión plenaria del Comité Central del Partido Comunista de China (PCCh) celebrada en Beijing del 26 al 29 de octubre de 2015, constituye una referencia decisiva en un periodo clave del proceso de reforma y apertura. Tanto por su contexto como por su contenido y expectativas, está llamado a ser un documento clave en la larga transición china.

Cabe señalar, de entrada, que a pesar del incremento sostenido del papel del mercado como consecuencia del proceso de reforma, la planificación de las políticas de desarrollo, iniciadas en 1953, constituye una señal de identidad irrefutable del modelo económico y político chino.

Este XIII Plan Quinquenal debe tener en cuenta, al menos, tres claves determinantes. En primer lugar, el hecho de que en el transcurso del próximo lustro China se convertirá, cualquiera que sea el método de medición que utilicemos, con toda probabilidad, en la primera economía del mundo, una circunstancia que tendrá un singular impacto en la economía global y de la que devendrá una implicación mayor en las cuestiones relativas a la gobernanza en todos los sentidos.

China es ahora la segunda mayor economía del mundo y el mayor exportador. En diciembre de 2014, el yuan superó al euro para convertirse en la segunda divisa, tras el dólar, más utilizada en la financiación del comercio mundial. De aquí a 2020, China se convertirá en el mayor inversor internacional del mundo. La internacionalización del renminbi (RMB) será uno de los ejes determinantes del próximo lustro. Sus activos globales se triplicarán, pasando de 6,4 billones a 20 billones de dólares, estiman fuentes autorizadas. Todo ello contribuirá a fortalecer su posición económica global.

En segundo lugar, la aceleración del proceso de transformación estructural que vive la economía china, significando el mayor papel atribuido a la economía privada en la modernización, al igual que el papel del mercado en contraposición con el aligeramiento de la burocracia y el ajuste del sector público. Por otra parte, la reforma rural debe experimentarunareestructuración de gran alcance para ganar eficiencia. Igualmente, el gasto en $\mathrm{I}+\mathrm{D}$, que en 2014 ascendió al 2,1 por ciento del PIB (en Shanghai, 3,6 por ciento), seguirá en ascenso para convertir a China en una potencia tecnológica. En lo ambiental, los esfuerzos deben adquirir una notoria visibilidad dado el estado del país en este aspecto. Por otra parte, debe dar cabida a una ambiciosa reforma del sector público para crear gigantes industriales capaces de enfrentarse a los grandes competidores privados mundiales, complemento 
del plan Hecho en China 2025 que debe contribuir a posicionar globalmente sus propias marcas comerciales. Asimismo, debe dar cabida a ambiciosos proyectos como la Franja y la Ruta y los corredores económicos, proyectos que cuentan con numerosos fondos adscritos al igual que otros de signo bilateral habilitados con terceros países y que deben generar nuevos mercados para las ínfulas productivas chinas, al amparo de los nuevos instrumentos crediticios instituidos.

En tercer lugar, el acoplamiento de todos los eslabones de la reforma, más allá de la significación de lo estrictamente económico, atendiendo a los aspectos que inciden en la plasmación del objetivo de construcción de una sociedad acomodada. La creación de un moderno sistema de asistencia social que atienda a las características del país debe contribuir de forma decisiva a la superación de una penosa desigualdad que sigue ensombreciendo los efectos de la gaige-kaifang.

Todo ello sin menoscabo de los factores políticos, especialmente los relacionados con el fortalecimiento de la legalidad y el Estado de derecho, dando un nuevo impulso a la construcción normativa. Ese proceso tiene su reto clave en el arbitrio de una nueva cultura de relación entre el poder y la sociedad.

En suma, el XIII Plan Quinquenal, que alcanzará a la víspera del centenario del PCCh (2021), determinará el rumbo del proceso de modernización.

\section{Unos planes con historia}

La tradición de los planes quinquenales en China se remonta, claro está, a los inicios del maoísmo, durante el período de influencia soviética, con el PCCh ya en el poder (1949). En 1953 se lanzó el primero, de la mano del legendario Chen Yun, más tarde víctima de la Revolución Cultural y recuperado después por Deng Xiaoping tras la muerte de Mao. La combinación de la influencia planificadora leninista con esa cultura estratégica china que apunta a una visión que tiene siempre una mano en el presente y otra en la historia, permitió en buena medida que, en pocas décadas, China pudiera recuperar una parte sustancial de la grandeza perdida durante el periodo de decadencia iniciado esencialmente en el siglo XIX. En coherencia con ese legado, la agenda actual china tiene ya la vista puesta en dos fechas "inmediatas": 2021 (centenario del PCCh) y 2049 (centenario de la República Popular China). Este XIII Plan es inseparable de dichos tiempos.

Dos primeras claves habría que tener en cuenta a la hora de valorar el documento. Primero, la transición hacia un nuevo modelo de desarrollo. Ya en tiempos de Hu Jintao (2002-2012) se tomó conciencia de que el modelo iniciado 
en 1979 estaba agotado. La combinación de mano de obra barata, inversión a gran escala y orientación de la producción hacia el exterior permitió un salto gigantesco a la economía china, pero los efectos secundarios no fueron desdeñables. El XVIII Congreso del PCCh (2012) ratificó el nuevo rumbo, incorporando especialmente las dimensiones ambientales y tecnológicas y propiciando mudanzas estructurales de gran calado con vistas a lograr el cambio de carril, aun a precio de reducir la velocidad de la locomotora.

Segundo, la construcción de una sociedad acomodada. Se ha reiterado hasta la saciedad que el éxito del proceso chino y la ausencia de grandes conflictos sociales que lo desestabilizaran (el más grave fue la crisis de Tiananmen en 1989) se explica por un pacto no escrito que ofrece a la ciudadanía riqueza a cambio de lealtad. Pero esta ecuación también agotó su recorrido. La sociedad china es más rica en su conjunto pero tremendamente desigual. Las propias autoridades reconocen un índice Gini de 0,469, por encima del umbral de alerta del 0,4. No falta quien relativice estas cifras y las eleve al 0,6. En cualquier caso, la culminación del proceso de modernización del país y su entronización en la cima de la economía global, superando incluso a EEUU en términos absolutos, no podrá lograrse con una sociedad fracturada y con unos índices de desigualdad insostenibles. Esto requiere grandes inversiones en salud, educación, mejora de las infraestructuras rurales, urbanización, nuevas políticas demográficas, etc. Y, por supuesto, un aumento sustancial de los salarios para crear esa clase media que dé soporte a la sociedad de consumo y aliente el desarrollo de los servicios en detrimento de los sectores secundario y primario.

Pero como es obvio, el Plan Quinquenal no es solo economía. Y en China, sus fronteras con la política son en extremo vagas. En primer lugar, porque del éxito económico de la reforma depende el propio futuro del PCCh. Y esto explica el vigor de la lucha contra la corrupción y los cambios introducidos en el modelo de gobernanza con vistas a crear un Estado con derecho, donde la norma pese más que la decisión de los funcionarios del partido, tantas veces arbitraria. En segundo lugar, a la regeneración interna se suma esa segunda transformación en el aparato del Estado, propiciando una significativa mutación de la burocracia que parte de la promoción de una mayor autonomía y un robustecimiento de su condición de servicio a la ciudadanía, extremos esenciales y largamente dilapidados en el largo proceso de confusión de las últimas décadas que favoreció la práctica superposición del Partido y el Estado. Aún así, que nadie se imagine separaciones radicales de ambos actores.

El sentido general de los cambios que vive China en la actual fase de la reforma tiene un denominador común cualitativo que no es baladí. La apuesta por aumentar el papel del mercado, de la economía privada, la mitigación de los monopolios, etc., es de gran alcance. La primera consecuencia será 
la reducción progresiva del peso del sector público en el conjunto de la economía, liberando fuerzas que hasta ahora el PCCh mantenía a buen recaudo. La transformación de esa realidad podrá suponer una reducción del poder del PCCh cuyas capacidades de intervención son aun de gran calado. Lo vimos durante la crisis bursátil de este verano. Otra consecuencia podría llegar a ser la experimentación de un diálogo inédito y de nuevo tipo entre el PCCh y las nuevas fuerzas emergentes, hoy claramente asimétricas.

¿Hasta donde podrá llegar el PCCh con la liberalización de la economía y la preservación de su hegemonía política? Los dirigentes actuales consideran que aún hay margen para una liberalización que no cuestione su liderazgo. Y la necesitan para conseguir sus objetivos históricos. Pueden asumir más mercado, más economía privada, pero también una democracia capaz de preservar su poder. Para ello precisa evitar que las nuevas realidades económicas se traduzcan en grupos de presión con vocación desafiante. Hasta ahora lo ha conseguido. No está claro que pueda seguir siendo así en un contexto de reducción progresiva de su poder económico.

¿Tienen sentido los planes quinquenales hoy día? En virtud de su realidad económica y política, sin duda, así es y resultan de gran utilidad. Otra cosa es que su protagonismo tienda a la baja a medida que el cambio en el modelo económico se acentúe y emerjan otros actores que pongan sobre la mesa otros modos de operar. Será entonces cuando el PCCh deberá demostrar que dispone de la cintura suficiente para gestionar los nuevos equilibrios.

En cualquier caso, el Plan Quinquenal forma parte del modus operandi de la gestión de la economía nacional en China, lo cual implica la definición de las prioridades nacionales y de la orientación del desarrollo económico y social a medio y largo plazo. A excepción del periodo comprendido entre 1949 y 1952 y entre 1963 y 1965, China ha contado siempre con planes quinquenales.

El I Plan Quinquenal (1953-1957), gestado con la ayuda soviética, se orientó a la construcción del sistema industrial básico. De la mano de Chen Yun, se establecieron los vectores esenciales de la transformación socialista de la economía, si bien hoy día se considera en su debe un ritmo demasiado apresurado e intenso, lo cual dejó secuelas durante un periodo bastante largo.

El II Plan Quinquenal (1958-1962) concretó los principales índices, con objetivos muy ambiciosos, que de continuo fueron objeto de revisión al alza como consecuencia de la influencia del pensamiento del "avance temerario". El movimiento del "Gran Salto Adelante" provocó grandes desequilibrios en la economía nacional que derivaron en una grave crisis condicionada por la escasez de alimentos, la sobreproducción de objetos de baja calidad. El reajuste llegó en 1961 con la llamada "restauración burocrática".

El III Plan Quinquenal (1966-1970) trató de abordar los principales retos de la economía china en el marco de las "cuatro modernizaciones" y en 
un contexto político nuevamente adverso condicionado en primer lugar por la política estratégica de prepararse para la guerra y las calamidades naturales, y, posteriormente, tras el anuncio de la Gran Revolución Cultural que no impidió la ciega persecución de la alta velocidad y alta acumulación, enfatizando el autoabastecimiento como referente de la construcción de la economía nacional, determinando el cumplimiento de dicho plan.

El IV Plan Quinquenal (1971-1975), tuvo un reajuste en 1973 ante el delicado cariz de la economía, en tiempos aun muy convulsos internamente pero con clara tendencia a la normalización, lo que permitió cierto repunte.

El V Plan Quinquenal (1976-1980) se insertó en el Esquema de Proyecto de Diez Años para el Desarrollo Económico (1976-1985), en tiempos marcados por la recuperación económica pero también por esa sempiterna obsesión de acelerar el paso de la construcción económica al precio que sea, lo cual propició efectos indeseables. La III Sesión Plenaria del XI Comité Central del PCCh, que marcó el inicio de la reforma y apertura, enfatizó la necesidad de gestionar la economía atendiendo a sus leyes objetivas y no al voluntarismo, además de experimentar de forma continua con propuestas innovadoras con la preocupación común de promover el desarrollo económico con cambios añadidos en el orden social al dar máxima prioridad a la modernización.

El VI Plan Quinquenal (1981-1985) ya incorporó objetivos orientados al final del siglo XX, definidos con técnicas más solventes y rigurosas, encauzando el rumbo de la economía nacional con metas y exigencias concretas cuyo cumplimiento fue mundialmente reconocido. El PIB creció a una media anual del 11 por ciento durante este periodo.

El VII Plan Quinquenal (1986-1990) enfatizó la importancia de reformar la estructura económica en el marco de un plan de desarrollo económico y social que fue el primero de su tipo en la historia de la economía planificada de China. La tasa media de crecimiento anual fue del 7,9 por ciento.

El VIII Plan Quinquenal (1991-1995) permitió adelantar en un lustro la consumación del objetivo histórico de cuadriplicar en 2000 el PIB de 1980. En dichos años, la reforma de la estructura económica logró avances importantes, con un sobresaliente desarrollo del comercio exterior. El crecimiento medio anual durante la vigencia de este plan fue del 11 por ciento.

El IX Plan Quinquenal (1996-2000), a caballo entre dos siglos, se complementó con la fijación de las metas a largo plazo para 2010, destacando ambiciosos objetivos en materia de PIB pero también demográficos, sociales, reforma de las empresas estatales o formación de un sistema con importancia creciente del mercado. La tasa de crecimiento medio anual fue del 8 por ciento.

El X Plan Quinquenal (2001-2005) materializó cambios significativos en el orden socioeconómico. El PIB de China superó los 1,6 billones de dólares 
y el PIB per cápita superó los 1.000 dólares. La presencia internacional del país aumentó de forma significativa.

El XI Plan Quinquenal (2006-2010) apunta ya a un cambio de tendencia que se ve afectado por el estallido de la crisis financiera global (2008), lo cual obliga a reaccionar con la habilitación de paquetes de estímulo no contemplados inicialmente. Es un lustro de zozobra y expansión en el cual China, al tiempo que se exhibe como una potencia industrial capaz de arrebatar el liderazgo a EEUU en este terreno, toma nota de los visibles agujeros y carencias de su nueva grandeza, especialmente en los órdenes social, ambiental o tecnológico, lo cual plantea la inexcusable incorporación de estas variables a los planes de desarrollo del país.

\section{Una aproximación al XII Plan Quinquenal}

El XII Plan Quinquenal(2011-2015) fue planteado como un ejercicio crucial para construir esa sociedad próspera y sostenible con base en la profundización de la reforma. Su marco subyacente fue la "sociedad armoniosa" que formuló $\mathrm{Hu}$ Jintao, aspirando a reducir las diferencias de ingreso entre el campo y la ciudad, las regiones del Este y el Oeste y entre ricos y pobres. Igualmente se planteó conceptualmente la corrección de los desequilibrios acumulados en los últimos 30 años. Y planteó el objetivo final de evolucionar desde un modelo de crecimiento cuantitativo a otro cualitativo, superando el modelo predominante en las últimas décadas a través de la promoción de un "desarrollo científico". El objetivo de crecimiento de este plan se cifra en el 7 por ciento anual.

El balance este XII Plan Quinquenal debe otorgar especial relevancia a los avances registrados en materia de infraestructuras y también a la modernización de la agricultura, ambos muy notorios, pero igualmente al orden social, especialmente en materia de ingresos tanto de asalariados como pensionistas, tanto en el campo como en la ciudad, aunque persistan aun grandes diferencias.

El crecimiento promedio del XII Plan Quinquenal fue del 7,8 por ciento, cifra muy superior al promedio mundial del 2,5 por ciento. En cualquier caso, pese a dicha cifra, ha sido un lustro marcado por el debate sobre un hipotético aterrizaje forzoso de la economía china y cierta sensación de colapso. Cabe recordar que en el tercer trimestre de 2015, la economía china creció un 6,9 por ciento, siendo la primera vez que el crecimiento trimestral disminuyó por debajo del siete por ciento desde el segundo trimestre de 2009 , en pleno auge de la recesión global.

Pese a todo, los datos, sin embargo, confirman que China es ya la segunda economía mundial con un valor superior a los 10 billones 
de dólares y aporta más de la cuarta parte del crecimiento económico mundial, desempeñándose como número uno en los sectores manufacturero y comercial, con una tasa de urbanización que superó el 50 por ciento. Progresivamente, en estos años, ha pasado de ser símbolo de la exportación de pequeños bienes de consumo a exportar trenes de alta velocidad. Por otra parte, sigue siendo uno de los países receptores de inversión más atractivos.

Esto no obsta a reconocer que China vive una situación nueva, la "nueva normalidad" según la terminología oficial, caracterizada por un crecimiento económico más lento y, necesariamente, más equilibrado y sostenible. Esa baja en el ritmo es indispensable para llevar a buen término la reestructuración económica y el cambio en el modelo de desarrollo, asociándolo más a la innovación y la calidad que a las inversiones y exportaciones.

Todo este periodo ha estado marcado por el condicionante de la reforma, erigida en palabra clave de este lustro con aplicación de medidas en diversos órdenes, ya hablemos de las empresas estatales, el sistema fiscal o financiero o la administración. En 2013 se construyó en Shanghai la primera Zona de Libre Comercio, concebida como lugar de prueba para promover una mayor apertura de la economía.

También se lanzó la iniciativa de la Franja y la Ruta con el propósito de impulsar la construcción de infraestructura y la cooperación financiera, entre otros, a lo largo de tres continentes (Europa, Asia y África), con instituciones añadidas como el BAII (Banco Asiático de Inversión en Infraestructuras).

En el ámbito de la gestión, las autoridades rebajaron el número de aprobaciones administrativas para ensanchar el espacio del mercado, afanándose por fomentar la creatividad y el emprendimiento empresarial.

En suma, alejándose poco a poco del modelo anterior propiciando el avance hacia un modelo impulsado por el consumo.

La lucha contra la corrupción, como expresión de la afirmación del Estado de derecho, es signo igualmente de esta etapa. Más de la mitad de los miembros del Comité Central del PCCh elegidos en el XVIII Congreso (2012) fueron desplazados o separados de sus cargos. Se trata de un cambio a gran escala poco habitual en la historia del Partido e inseparable del tono, intenso, de la lucha anticorrupción. Un total de 104 de los 205 miembros del Comité Central fueron promovidos, separados o expulsados de sus cargos desde 2012. Entre ellos, 81 fueron promovidos, 16 fueron transferidos a puestos menos importantes (en su mayoría se trata de funcionarios mayores de 65 años) y siete fueron separados.

La campaña anticorrupción afectó a 7 miembros del Comité Central, incluyendo a Ling Jinhua (ex director del Departamento de Trabajo del Frente Unido), Zhou Benshun (ex jefe del Partido en Hebei) y Yang Dongliang (ex responsable de seguridad laboral). Más de 100 funcionarios de nivel 
ministerial o superior fueron llevados a la justicia en el transcurso de la campaña iniciada en 2012 y cientos de funcionarios provinciales y locales fueron objeto de investigación, procesamiento y condena.

\section{Algunas claves del XIII Plan Quinquenal}

La trayectoria de elaboración de los planes quinquenales acredita esa creencia china de que el sistema económico debe fundamentarse en la planificación desde el Estado. Aun así, desde los tiempos de Chen Yun, quien consideraba la planificación como un mecanismo para controlar el sector privado, a día de hoy, el cambio ha sido significativo. Si bien no puede hablarse de equidad entre ambos sectores, lo cierto es que la economía privada es considerada hoy día un pilar sustancial de la economía nacional.

A diferencia de los primeros tiempos de la planificación, el PCCh no solo no quiere ahogar a la economía privada y otorgarle cierto margen de maniobra sino en línea con la evolución de su consideración, transformarla en un activo para el crecimiento y el desarrollo del país.

A propósito del XIII Plan Quinquenal, cabría destacar las siguientes claves:

a) Un enfoque centrado en la cuestión social, a fin de proporcionar mayores garantías de equidad y de justicia distributiva. El objetivo de construir una sociedad modestamente acomodada en 2020 incluye la satisfacción del compromiso establecido en el XVIII Congreso del PCCh (2012) de duplicar el ingreso per cápita en las zonas urbanas y rurales en comparación con los niveles de 2010.

b) Un enfoque centrado en el alivio del control estatal, reforzando el papel decisivo del mercado en la economía. Ello sin perjuicio del papel público en el desarrollo de las inversiones en áreas clave como las infraestructuras y la mejora de la dirección macroeconómica.

c) Un enfoque centrado en la mejora del medio ambiente.

d) Un enfoque centrado en las reformas financieras y la internacionalización del yuan.

e) Un enfoque centrado en la definición de un crecimiento más sostenible, aunque más lento, dando paso a esa "nueva normalidad" con el impulso de la demanda interna y la innovación, auspiciando la alta tecnología como un sector industrial de primer orden.

f) Un enfoque centrado en la mejora de la gobernanza, poniendo el énfasis en la regulación sobre el gobierno limpio y reforzando la disciplina interna del PCCh, la democracia, el Estado de derecho y la 
independencia de la justicia, todo ello sin alterar de forma significativa los límites del sistema político vigente.

Se trata, en suma, de establecer las bases para acelerar la reforma integral y el cambio histórico que constituye la esencia del sueño chino. Las necesidades a abordar sugieren que el próximo período será de los más complejos de la reciente historia china, tanto en razón de sus circunstancias internas como externas. Su probable ascenso a la supremacía económica global se contextualiza en el marco de los imperativos derivados de los acuerdos comerciales como el TPP o el TTIP a los que opone respuestas, en primera instancia, basadas en el fomento de los TLCs y de plataformas alternativas como la RCEP.

\section{El contexto del XIII Plan Quinquenal}

Desde el primer trimestre de 2011, el crecimiento chino está en baja constante; en cuatro años, perdió cuatro puntos. El freno al elevado ritmo de crecimiento es un hecho incontestable, al igual que las diversas medidas orientadas a su relanzamiento, desde reducciones de las tasas de interés (seis en lo que va de año) acompañadas de una nueva rebaja en la tasa de reserva obligatoria, además del levantamiento del control de la tasa de remuneración de los depósitos, medida largamente prometida, autorizando la competencia entre los bancos.

Pero si en otro tiempo podría preocupar una tasa del 6,9 por ciento, a priori débil en función de los parámetros chinos, hoy no es así porque China se está instalando en otro paradigma que promueve con clara determinación, tanta como riesgos incorpora, ya sea en el plano estrictamente económico o político.

Cuando estalló la crisis financiera de 2008, China reaccionó con un vasto plan de inversiones. Algunas informaciones apuntan a que entre $2009 \mathrm{y}$ 20012 se inyectaron en la economía el equivalente a unos 600 mil millones de dólares. Esa maniobra funcionó. Entre el segundo trimestre de 2009 y el primer trimestre de 2010, el crecimiento pasó de 7,9 por ciento a 11,9 por ciento. Pero tuvo también algunos efectos perversos. El primero fue la moderación de la transición para primar la atención a dicha urgencia. Segundo, el despilfarro de recursos, cierto descontrol de las administraciones provinciales y de los bancos conectados a los grandes grupos públicos, principales responsables de las deudas tóxicas.

Ese retraso en las reformas combinado con la atonía de los mercados de exportación y una serie de factores internos empujaron el crecimiento a la baja. En el cuarto trimestre de 2010, ascendía al 9,8 por ciento y 
en noviembre de 2012, cuando llegó al poder el nuevo equipo dirigente, caía al 7,8 por ciento. Desde entonces no cesó de bajar. Las nuevas autoridades responden entonces con medidas de estímulo, pero más sutiles que las adoptadas en 2008, emulando en cierta medida el comportamiento de las autoridades japonesas, inglesas o estadounidenses en atención a sus propias circunstancias y necesidades.

Desmintiendo los análisis más pesimistas sobre la marcha de las reformas y su lentitud, otras fuentes resaltan lo avanzado en estos dos últimos años con una transición claramente en curso que perfila poco a poco las líneas maestras de las orientaciones básicas y asegura su implementación experimental y progresiva. Los signos de ese desplazamiento vendrían dados por el vigor de su sector financiero -a pesar de los trances de la bolsa en el verano último-, o el dinamismo de los servicios y del consumo que aumentan su proporción en el conjunto de la economía. Si la producción industrial representaba en 2005 el 46,9 por ciento del PIB comparada con el 41,4 por ciento de los servicios, en 2012 , la parte de servicios ya sobrepasaba a la industria y las proyecciones para este año 2015 apuntan a un 49,2 por ciento frente al 41,9 por ciento de la industria. Los servicios son ahora la principal fuente de empleo en China.

La actividad de los servicios financieros progresó más del 16 por ciento en el tercer trimestre de 2015 en relación al mismo periodo de 2014, con cifras que doblan el progreso de la economía tradicional. La percepción de que el consumo, los servicios y un sector financiero más dinámico se afirman como nuevos ejes del crecimiento es ampliamente compartida, dentro y fuera de China.

\section{Los contenidos conceptuales del XIII Plan Quinquenal}

Las expectativas son altas y el PCCh se enfrenta a una tarea de enormes proporciones con un horizonte de reformas que deben ser sólidas y, a la vez, flexibles. Los ajustes estructurales no son palabas huecas sino exigencias reales a las que difícilmente puede sustraerse, a pesar de sus efectos, de entrada, no siempre positivos. Las grandes opciones estratégicas incluyen la activación de un nuevo impulso a la reforma y apertura al exterior, la integración de la economía regional y la identificación de nuevos motores del crecimiento.

Como ya se indicó, el objetivo central -el que quizás mejor resume las expectativas- arranca del XVIII Congreso del PCCh: el compromiso de construir al completo en 2020 una sociedad modestamente acomodada, lo cual requiere duplicar tanto el PIB como los ingresos per cápita en comparación con 2010. 
El ritmo del crecimiento económico, el cambio de modalidad de desarrollo y su actualización, la ejecución de la reforma en los sectores clave son ideas-fuerza que el grupo dirigente actual, en ejercicio desde noviembre de 2012 y atravesado el ecuador de su mandato, debe plasmar en políticas. El eje central de su pensamiento sigue siendo el desarrollo, concebido como tarea primordial. Traducido en porcentajes, el ritmo de crecimiento podría rondar el 6,5 por ciento, quizá algo más, cifras que permitirían alcanzar las dos metas citadas.

La clave de bóveda es, en cualquier caso, el cambio en el modelo de desarrollo, de forma que sin la mejora de la calidad y la eficiencia, con atención a los aspectos fiscal y tributario (reforma del sistema de gestión del presupuesto, de la estructura tributaria y de las relaciones entre el gobierno central y los gobiernos locales) y otras dimensiones estrictamente económicas difícilmente podrá consumarse con éxito.

Otro pilar sustancial será la planificación regional que debe orientarse a favorecer la modificación de la estructura económica y la mejora de la demanda doméstica. En este sentido, grandes proyectos como la Franja y la Ruta (que implica una dinamización al alza del Oeste del país), la franja económica de Beijing-Tianjin-Hebei (como pilar de la modernización administrativa regional) y la franja del río Yangtsé (concebida como columna vertebral de la economía china) son tres referentes del crecimiento que desempeñan un papel preeminente en este XIII Plan.

Se anuncian igualmente reformas profundas en el sistema financiero para establecer un mercado de capitales transparente, apoyando la inclusión del RMB en el paquete de derechos especiales de giro del FMI y promoviendo la liberalización de la cuenta de capital y la internacionalización del yuan.

También contempla la apertura de más sectores monopolísticos a la inversión en los próximos cinco años, en especial, petróleo, gas natural, electricidad, telecomunicaciones, transporte y servicios públicos. Y, complementariamente, el creciente estímulo a empresas chinas, con especial proyección del sector público hasta ahora, a invertir en el extranjero.

La mejora del medio ambiente es clave. En los próximos cinco años debiera experimentarse una ligera mejora en la protección ambiental como resultado de la implementación de los planes de acción contra la contaminación del aire y del agua. Se anuncia un Fondo de Desarrollo Verde para promover un modelo de producción limpia y un crecimiento sostenible, con el fomento de la energía limpia y el transporte verde, el control de las emisiones de carbono en las principales industrias y el impulso de la economía circular. Entre las medidas anunciadas cabe citar 
el envite a volver más independiente el órgano de control limitando las posibilidades de interferencia de otros órganos. Esto implicará la supresión de los servicios de medio ambiente de nivel distrital y en su lugar se establecerán agencias de supervisión. El cambio será gradual, reforzará las capacidades de los departamentos provinciales de medio ambiente y se llevará a cabo con experiencias piloto previas. Todo ello se enmarca en una revolución energética que contempla reducir el consumo de energía, agua y terrenos para construcción y su consumo por unidad de PIB, acelerando también la sustitución del uso del carbón y otros combustibles fósiles. Las emisiones de carbono bajaron un 2 por ciento en 2014 por primera vez desde 2001. China no tiene otro camino responsable.

El campo, tantas veces olvidado, sigue representando un valor clave de la economía y la estabilidad de China. La mejora de la gestión de la tierra sugiere la planificación integral de su uso y matizar la explotación comercial, así como el otorgamiento de más poder, responsabilidad $\mathrm{y}$ beneficios a los distritos y condados para dinamizar la reforma $\mathrm{y}$ ensayar alternativas de aprovechamiento. Un plan de reforma rural con el doble objetivo de elevar los ingresos de los agricultores y proseguir la modernización del sector es objeto de ultimación. Para 2020, los sistemas de gestión agrícola y de derechos de propiedad colectiva rural se modificarán con programas piloto.

Uno de los anuncios estrella tras la sesión plenaria de octubre fue el relativo al abandono de la política del hijo único. Según las tendencias demográficas actuales, China tendrá en 2015, un déficit de 15 millones de personas respecto a lo planeado. Ese descenso está relacionado con una tasa de mortalidad más elevada y un deseo de fertilidad estancado. De seguir así las cosas, en 2020, el déficit ascendería a 50 millones de personas. Es por ello que la política de control demográfico debe relajarse, aunque puede necesitar, por añadidura, medidas de estímulo y apoyo a las familias. Las tornas se han invertido.

Otro de los anuncios fue la extensión de la pensión de vejez a toda la población, adoptando un esquema de seguro social más equitativo y sostenible capaz de abarcar al conjunto de los ciudadanos, con un seguro de enfermedad a escala total que iguale el tratamiento de residentes urbanos y rurales. Complementariamente, el gobierno se propone ampliar los canales de inversión de los fondos de seguridad social. Cerca de 200 millones de chinos aun están pendientes de ser incluidos en el esquema de seguro social para ancianos del país, según cifras oficiales. En septiembre de 2014, unos 650 millones de un total de 1.300 millones de chinos estaban cubiertos por un seguro de enfermedad grave. Quienes más se han sacrificado por generar la prosperidad de la que China hoy disfruta tienen 
sobrado derecho a una redistribución equitativa de los beneficios. Esto demanda sacar a toda la población rural de la pobreza en 2020, reduciendo en más de 600 la lista de distritos catalogados oficialmente como pobres.

El XIII Plan no olvida las estrategias regionales y una mayor integración de Internet en la industria tradicional. Esta dimensión incluye la formación de franjas económicas a lo largo de los ríos, autopistas y vías férreas importantes, así como en regiones costeras, prestando especial atención a diversas áreas económicas clave. El Plan Internet Plus, por su parte, quiere volver más eficientes las industrias obsoletas.

Finalmente, la innovación tecnológica apunta también al desempeño de un papel líder en proyectos científicos internacionales, no solo al fortalecimiento de las capacidades internas a este nivel. Durante los primeros nueve meses del presente año, el sector de alta tecnología experimentó un crecimiento del 10,4 por ciento en la producción de valor agregado, es decir, 4,2 puntos porcentuales por encima de la tasa del sector industrial al completo. La posición de la innovación se eleva a la condición de clave en el desarrollo económico. Es proceso es inseparable de la promoción del ascenso del sector industrial hacia la gama media superior.

La tasa de urbanización debe crecer a un ritmo más rápido. La proporción de residentes urbanos registrados con respecto a la población total fue del 35,9 por ciento a finales de 2014. El plan nacional prevé elevar la proporción al 45 por ciento.

\section{Las expectativas}

En la tradición china, cada 60 años señalan un círculo, así que el año 2016 será el comienzo de un nuevo círculo.

Xi Jinping alude a las "tres leyes" y los "tres desarrollos": un camino de desarrollo científico que sigue las leyes de la economía, un camino de desarrollo sustentable que sigue las leyes de la naturaleza, y un camino de desarrollo inclusivo que sigue las leyes de la sociedad humana. Y lo resumió en cinco ideas: innovación, coordinación, medio ambiente, apertura y colaboración. La Conferencia Central de Trabajo Económico, en diciembre, debe dar más pistas sobre el desarrollo de estos preceptos y acerca de cómo equilibrar la necesidad de un crecimiento continuo con el imperativo para las reformas que alteran los incentivos tradicionales a favor del crecimiento.

Este plan debe significar, en primer lugar, un salto real en las condiciones de vida de los ciudadanos chinos. Lo sustancial es diseñar una modernización que sustente ambiciosas políticas sociales, que permitan que el desarrollo 
redunde en una mejora del bienestar general, posibilitando que la sociedad pueda recoger ahora los frutos de su esfuerzo a lo largo de las pasadas décadas en condiciones verdaderamente gravosas.

Por otra parte, continuará la reforma hacia una economía de mercado para que este desempeñe un papel más esencial, con una reducción significativa y creciente de las interferencias del gobierno en las actividades económicas, incluyendo la fijación de precios. Tener en cuenta que el mercado está llamado a jugar un papel decisivo en la distribución de los recursos en un contexto en que el capital ejerce una influencia cada vez mayor se debe equilibrar con que el plan garantice que el mercado no perjudique el bien común.

El crecimiento en los próximos años no puede ser inferior al 6,6 por ciento para alcanzar los objetivos generales propuestos. Se trata de un crecimiento moderado en el contexto chino pero exigible para combinar la calidad del crecimiento con las expectativas. Aun así, el XIII Plan transmite menos preocupación por los índices mientras acentúa un cambio radical que se viene gestando en los últimos años, concediendo primacía a metas cualitativas, ya hablemos de la optimización industrial, el desarrollo tecnológico, la coordinación regional, sustentados en iniciativas y planes que tienen sus raíces en documentos previos, actualizados ahora con nuevas expresiones (Hecho en China 2025, la Franja y la Ruta, etc.), pero con el denominador común de lograr ese desarrollo de mejor calidad, más eficaz, sostenible y justo.

El XIII Plan Quinquenal será el primero desde que la economía china entró en el tiempo de la "nueva normalidad". Por ello, abrirá paso a una nueva ronda de apertura y acelerará el tránsito para transformarse en una economía abierta. Algunas provincias y ciudades han experimentado algunas reformas. Se establecieron cuatro zonas piloto de libre comercio en Shanghai, Tianjin, Fujian y Cantón, basadas en un modelo de gestión de trato nacional preestablecido y lista negativa. Beijing también abrió a los inversores extranjeros seis sectores de servicios que incluyen las finanzas, turismo y la salud. Estos procesos serán objeto de análisis, ponderación y generalización.

China está inmersa en una gran transformación, agravada por la enorme complejidad de su economía y su vulnerabilidad a los impactos externos. Las autoridades han hecho progresos en cuatro rubros: corrupción, medio ambiente, exceso de deuda de los gobiernos locales y exceso de capacidad.

El corazón de este plan afecta a la gestión de la transición económica que pilota el grupo dirigente para la profundización de las reformas que lidera el propio Xi Jinping. Esto implica una tarea titánica afectando a la reforma de los bancos públicos y de los grandes grupos industriales del Estado, pilares hasta ahora de un esquema de crecimiento considerado problemático, dilapidador de recursos y contaminante. La ofensiva está en curso y podría acelerarse. 
Uno de los problemas añadidos puede ser el aumento de la resistencia de la burocracia a la hora de tomar medidas audaces, trascender los intereses creados y mitigar los bloqueos al cambio.

La transformación de la economía china tendrá efectos sobre terceros países ya que un grupo más numeroso de chinos con ingresos medios generará más demanda de bienes y servicios extranjeros. Habrá un efecto derrame. El FMI prevé que la economía china será un 44 por ciento más grande en 2020 en comparación con el momento actual.

En suma, el XIII Plan Quinquenal resume grandes ambiciones, claramente inseparables de la realización del sueño chino. 


\section{Referencias bibliográficas:}

AAVV. (2006), El milagro chino visto desde el interior, editorial Popular, Madrid.

Au Loong Yu (2013), La Chine, un capitalisme bureaucratique, forces et faiblesses, Éditions Syllepse, París.

Bergère, Marie-Claire (2007), Capitalismes \& Capitalistes en Chine, Des origines à nos jours, Perrin Asie,

Bréhier, Delphine (2007), Miracles et revers de la croissance économique chinoise de 1949 à 2005, Le Manuscrit.

Gipouloux François (1993), La Chine vers l'économie de marché : une longue marche de l'après Mao. En Sociologie du Travail, Vol. 37, No. 1 (février 1995), pp. 107-110.

Lemoine, François (2006), L'Économie de la Chine, Collection Repères 378.

Lin Chun (2013), China y el capitalismo global. Reflexiones sobre marxismo, historia y política, El Viejo Topo, Barcelona.

Liu Suinian, Wu Qungan (1987), Economía socialista de China 19491984, Beijing Informa.

Ríos, Xulio (2007), Mercado y control político en China, La Catarata, Madrid.

Xue, Muqiao (1981), Problemas de la economía socialista de China, Ediciones en Lenguas Extranjeras, Beijing. 
